\title{
Genomic Exploration of Bacillus thuringiensis MORWBS1.1, Candidate Biocontrol Agent, Predicts Genes for Biosynthesis of Zwittermicin, 4,5-DOPA Dioxygenase Extradiol, and Quercetin 2,3-Dioxygenase
}

\author{
Adetomiwa A. Adeniji, ${ }^{1,2}$ Ayansina S. Ayangbenro, ${ }^{2}$ and Olubukola O. Babalola ${ }^{2, \dagger}$ \\ ${ }^{1}$ Human Metabolomics, Faculty of Natural and Agricultural Science, North-West University, Private Bag X6001, Box 269, \\ Potchefstroom, 2531, South Africa \\ ${ }^{2}$ Food Security and Safety Niche Area, Faculty of Natural and Agricultural Science, North-West University, Private Bag \\ X2046, Mmabatho 2735, South Africa
}

Accepted 4 February 2021.

\begin{abstract}
Many strains from Bacillus thuringiensis are known for their genomic robustness and antimicrobial potentials. As a result, the quest for their biotechnological applications, especially in the agroindustry (e.g., as biopesticides), has increased over the years. This study documents the genome sequencing and probing of a Fusarium antagonist (B. thuringiensis strain MORWBS1.1) with possible biopesticidal metabolite producing capacity from South Africa. Based on in vitro evaluation and in silico antiSMASH investigation, $B$. thuringiensis strain MORWBS1.1 exhibited distinctive genomic properties that could be further exploited for in planta and food additive production purposes.
\end{abstract}

Keywords: Bacillus thuringiensis, biocontrol, biopesticide, fungus-plant interactions, Fusarium, genome, genomics, in silico analysis, rhizospheric, secondary metabolites

Over the years, the biotechnological relevance of bacteria belonging to the genus Bacillus has been well documented. Their usefulness, particularly in the agricultural industry as biopesticides, biofungicides, bionematicides, and biostimulants (alternatives to synthetic fertilizers and chemical pesticides) has increased in the past decade (Adeniji et al. 2019b; DourietGámez et al. 2018; Egidi et al. 2016). Several strains from the genus (e.g., Bacillus thuringiensis) are producers of beneficial secondary metabolites (Chehimi et al. 2010; Daas et al. 2018) that have been exploited for plant biostimulation, biofertilization, and diverse biocontrol application (Azizoglu 2019; Sansinenea and Ortiz 2012). These secondary metabolites such as bacteriocins, chitinases, lipopeptides, and zwittermycin are attributed to

${ }^{\dagger}$ Corresponding author: O. O. Babalola; olubukola.babalola@nwu.ac.za

Funding: This work is based on the research supported by the National Research Foundation, South Africa (UID: 95111 France/SA bilateral); North-West University provided a post-doctoral fellowship for A. A. Adeniji.

The author(s) declare no conflict of interest.

(c) (1) () $\odot$ Copyright $(02021$ The Author(s). This is an open access article (c) 1 (1) 8 distributed under the CC BY-NC-ND 4.0 International license. be responsible for the antimicrobic and beneficial properties of the species (Daas et al. 2018; Hao et al. 2015; Hollensteiner et al. 2017).

In a previous investigation to identify candidate microbial agents for the control of fusariosis, a disease that disrupts major cereal grain trade globally (Adeniji and Babalola 2018) and is caused by phytopathogenic Fusarium spp. (e.g., Fusarium culmorum, $F$. verticilliodes, and $F$. graminearum), B. thuringiensis MORWBS1.1 was reported to exhibit antiFusarium properties (Adeniji et al. 2019a). To obtain the pure culture of the MORWBS1.1 B. thuringiensis isolate, $5 \mathrm{~g}$ of rhizosphere soil from a maize farm in the North West South African province was cultured on Bacillus HiCrome (Oxoid) selective agar based on the manufacturer's specifications. The isolate was molecularly and phylogenetically characterized as having antagonized $F$. culmorum and $F$. graminearium during an in vitro test on potato dextrose agar (Oxoid) - antagonism attributed to the bioactive metabolites produced by the isolate (Adeniji et al. 2019a). To further identify other beneficial biological properties of the strain in addition to its biofungicidal traits, we sequenced its genome.

The Zymo Research Soil-Microbe DNA Miniprep genomic isolation kit was employed for the extraction of the genomic DNA of isolate MORWBS1.1. MORWBS1.1 genome sequencing was carried out at Molecular Research DNA (Shallowater, TX, U.S.A.) using the Illumina MiSeq Reagent Kit v2 microsystem based on their internal protocols. The Nextera DNA sample preparation kit (Illumina) and Qubit double-stranded DNA highsensitivity assay kit (Life Technologies, Inc.), were employed for library preparation and final library concentration, respectively. To determine the library size, an Agilent 2100 Bioanalyzer (Agilent Technologies) was employed. This was followed by library pooling and dilution to $12.0 \mathrm{pM}$. Using a 600 -cycle v. 3 reagent kit (Illumina), having $12.0 \times$ average coverage, the paired-end reads were sequenced. The Kbase online platform (Arkin et al. 2016) was used for checking the quality of the reads (FastQC v.1.0.1), while reads trimming, gaps closing, and adaptor sequences removal were done using the Trimmomatic app (v0.32) and Cutadapt app (v1.0.1). The resulting sequencing reads were assembled using SPAdes (v3.13.0) on the KBase platform. The Kbase contigs were then uploaded on the NCBIProkaryotic Genome Automatic Annotation Pipeline (v4.2) 
(Pruitt et al. 2012), PATRICK online software server (v3.3.15) (Wattam et al. 2017), and RAST and SEED online server (v2.0) (Aziz et al. 2008; Overbeek et al. 2014) for annotation and comparative analysis. All of the bioinformatics analyses were performed with default settings.

The MORWBS1.1 whole-genome sequence yielded 6,112,031 bp and 92 contigs. The GC-content and $\mathrm{N}_{50}$ statistics were $34.72 \%$. and 220,282 bp, respectively. An aggregate of 6,411 coding genes was annotated (10 ribosomal RNAs [rRNAs], 42 transfer RNAs, eight crisper repeats, seven crisper spacers, and one crisper array). A statistical comparison of the MORWBS1.1 genome with other major $B$. thuringiensis strains is shown in Table 1. The analysis of the MORWBS1.1 subsystem is shown in Figure 1 and, from the subsystem coverage, 1,683 and 4,719 hypothetical and functional proteins, respectively, were identified. Furthermore, the subsystem feature counts also revealed six, 10, 12, and 53 features associated with sulfur, aromatic compounds, phosphorus, and iron acquisition metabolism, respectively. Other features include those associated with stress responses (43), regulation and cell signaling (33), and secondary metabolism (9). The antiSMASH (5.0.0rc1) (Blin et al. 2019) predicted 14 biosynthetic clusters and bioproducts (e.g., zwittermicin, fengycin, thuricin, polyxylopeptin, and bacillibactin) in the genome of strain MORWBS1.1. A comparison of the predicted MORWBS1.1 biosynthetic genes with other major B. thuringiensis strains is summarized in Figure 2.

The gene responsible for the synthesis of 4,5-DOPA dioxygenase extradiol, a core betalain biosynthetic pathway enzyme involved in the catalysis of betalain to betalamic acid (Christinet et al. 2004), was predicted in the MORWBS1 genome (Table 2; Fig. 3). Betalain has been reported to exhibit antioxidant and food additives properties (Adeniji et al. 2019b). Additionally, the gene responsible for the synthesis of quercetin 2,3-dioxygenase, a unique dioxygenase that relies completely on a mononuclear copper center for expression (Steiner et al. 2002), was predicted in the genome. Quercetin 2,3-dioxygenase catalyzes the insertion of molecular oxygen into polyphenol-flavonoids such as quercetin (antioxidant and dietary supplement) (Fusetti et al. 2002), which leads to the production of better degradable bioproducts (e.g., phenolic carboxylic acid ester derivatives) (Steiner et al. 2002) (Table 2; Fig. 4). At contig locations 11,656 to 12,739 (+ strand) and 7,950 to 9,930 (+ strand), genes for antifungal chitinase biosynthesis were identified (Table 2). The secondary metabolites identified in the MORWBS1.1 genome sequence makes it a suitable candidate for further biotechnological manipulation, particularly in the possible production of biopesticides and food additives, and in indigenous in planta biocontrol application in South Africa.

B. thuringiensis MORWBS1.1 was originally identified in 2017 and its 16S rRNA gene deposited in the GenBank with the accession number MF098612.1. Genome project data has been deposited at DNA Data Bank of Japan/European Nucleotide Archive/GenBank under the accession JAASGY000000000; GCA_011765855.1. The BioSample and BioProject designations for this project are SAMN11866291 and PRJNA544897, respectively.

Table 1. Statistical comparison of Bacillus thuringiensis MORWBS1.1 genome with other major B. thuringiensis strains ${ }^{\mathrm{a}}$

\begin{tabular}{lllccc}
\hline $\begin{array}{l}\text { B. thuringiensis } \\
\text { strain }\end{array}$ & \multicolumn{1}{c}{$\begin{array}{c}\text { GenBank ID; } \\
\text { assembly accession (NCBI) }\end{array}$} & Size (bp) & $\begin{array}{c}\text { G+C } \\
\text { numbers (\%) }\end{array}$ & $\begin{array}{c}\text { Number of } \\
\text { coding } \\
\text { Contigs }\end{array}$ & $\begin{array}{c}\text { Number } \\
\text { seq RNA } \\
\text { sences }\end{array}$ \\
\hline (rRNA, tRNA)
\end{tabular}

${ }^{a}$ Genome information for each strain is according to the data obtained from National Center for Biotechnology Information and Integrated Microbial Genomes \& Microbiomes system.

b Ribosomal RNA (rRNA) and transfer RNA (tRNA).
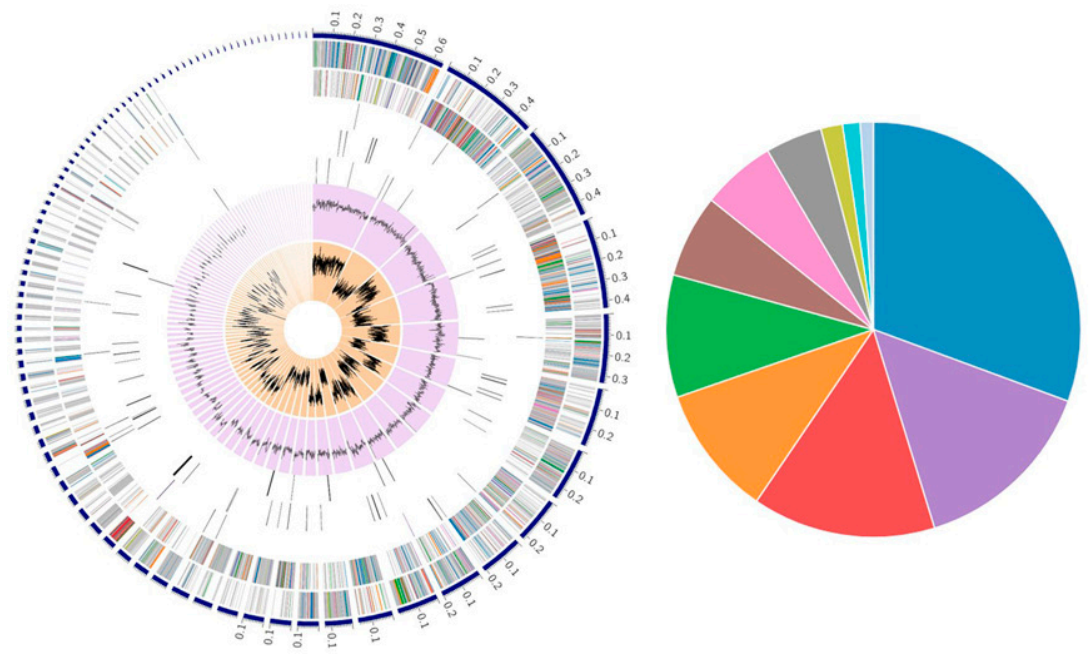

Bacillus thuringiensis MORWBS1.1

Subsystem (Subsystems, Genes)

METABOLISM (90, 801)

- STRESS RESPONSE, DEFENSE, VIRULENCE $(43,164)$

a PROTEIN PROCESSING $(42,243)$

- CELLULAR PROCESSES $(30,295)$

ENERGY $(28,289)$

DNA PROCESSING $(19,96)$

MEMBRANE TRANSPORT $(17,77)$

RNA PROCESSING $(13,60)$

- CELL ENVELOPE $(5,34)$

MISCELLANEOUS $(4,16)$

REGULATION AND CELL SIGNALING $(3,13)$

Fig. 1. Circular atlas of the distribution and subsystem summary of the Bacillus thuringiensis MORWBS1.1 genome annotations. Subsystem features from the outer to inner rings comprise contigs, coding sequence (CDS) (both forward and reverse strand), RNA genes, CDS (both antimicrobial resistance genes and virulence factors), $\mathrm{G}+\mathrm{C}$ content, and skew. Genomic features illustrated as the forward and reverse strand are depicted by the CDS colors (Wattam et al. 2017). 


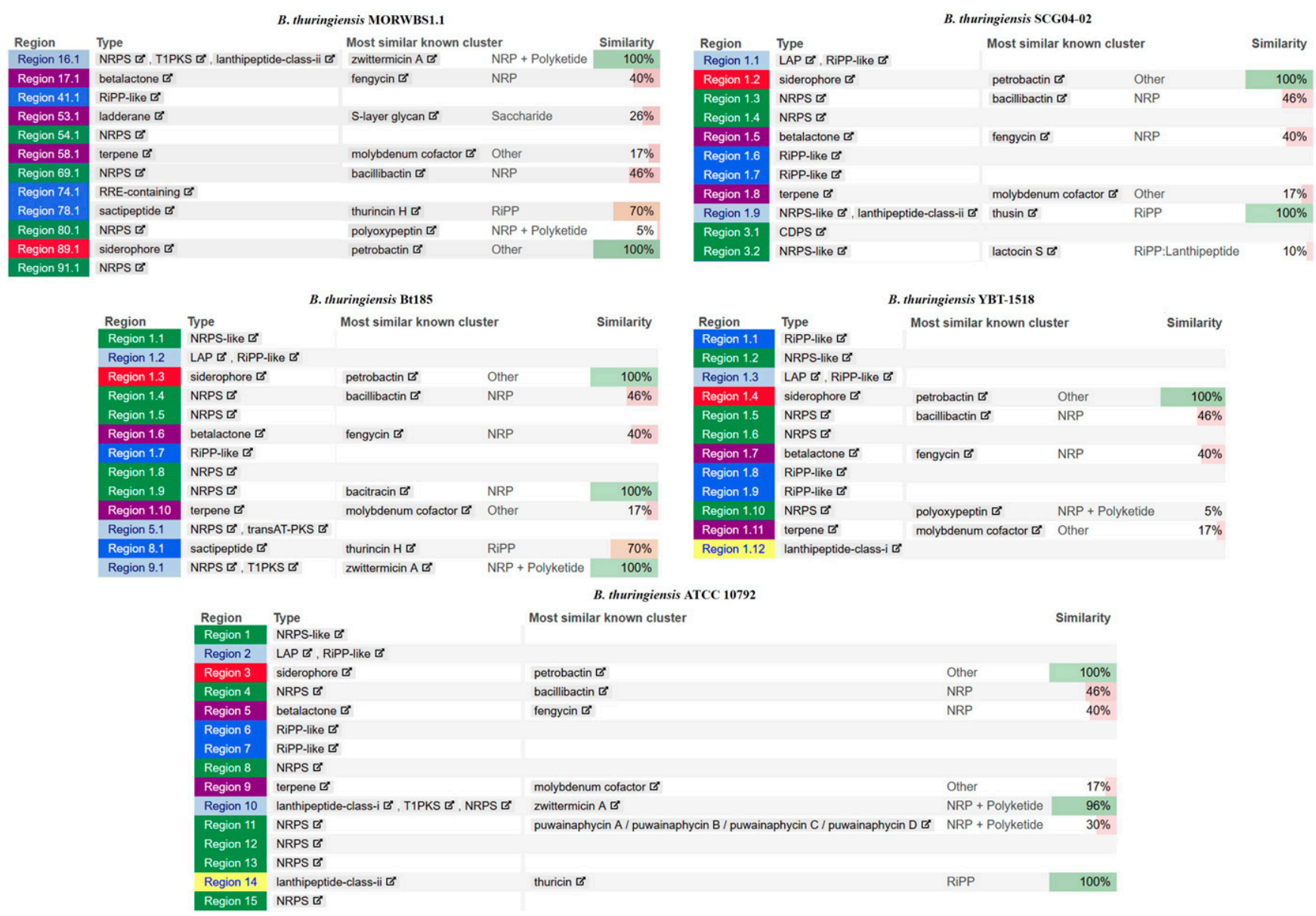

Fig. 2. Summary of the predicted MORWBS1.1 biosynthetic genes with reference to other major Bacillus thuringiensis strains.

Table 2. Notable genes predicted in Bacillus thuringiensis MORWBS1.1 genome

\begin{tabular}{lllll}
\hline Feature $^{\text {a }}$ & \multicolumn{1}{c}{ Product function } & Contig location & Protein length & DNA length \\
\hline Gene & Quercetin 2,3-dioxygenase & $196,518-197,217(+$ strand $)$ & 232 & 699 \\
Gene & 4,5-DOPA dioxygenase extradiol & $27,435-28,197(-$ strand $)$ & 253 & 762 \\
Gene & Zwittermicin A resistance protein ZmaR & $210,088-210,859$ (+ strand) & 256 & 771 \\
Gene & Chitinase A1 and D & A1: $7950-9,930(+$ strand) and & A1: 659 and D: 360 & A1: 1,980 and D: 1,083 \\
& & D: $11,656-12,739$ (+ strand) & & \\
\hline
\end{tabular}

\footnotetext{
${ }^{a}$ Coding sequence type.
}

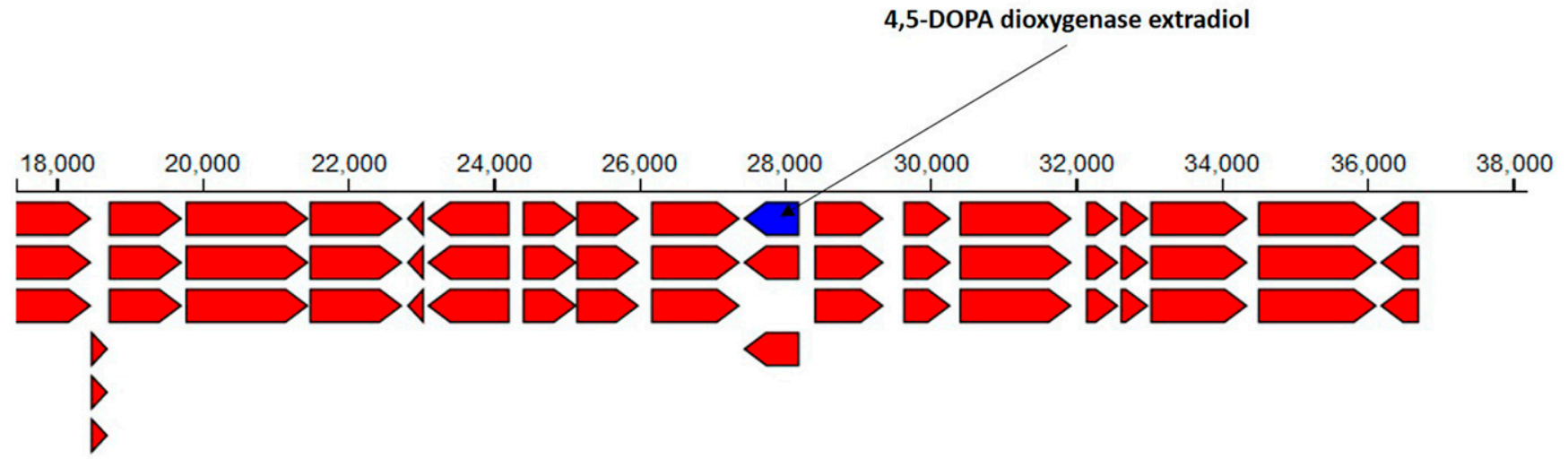

Fig. 3. Location of the predicted gene for the biosynthesis of 4,5-DOPA dioxygenase extradiol enzyme. 


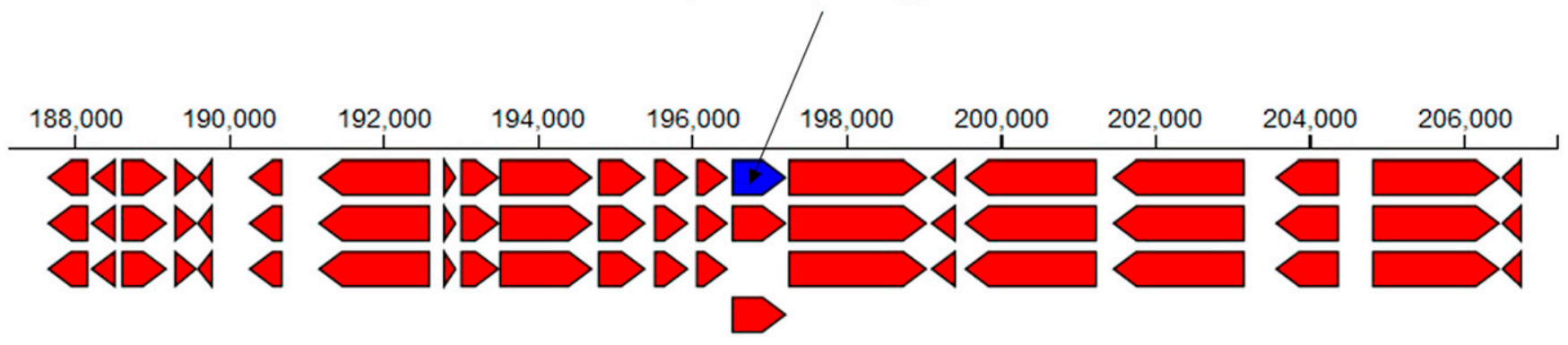

Fig. 4. Location of the predicted gene for the biosynthesis of quercetin 2,3-dioxygenase enzyme.

\section{AUTHOR-RECOMMENDED INTERNET RESOURCES}

DNA Data Bank of Japan/European Nucleotide Archive/GenBank: https://www.ncbi.nlm.nih.gov/assembly/GCA_011765855.1

BioSample:

https://www.ncbi.nlm.nih.gov/biosample/SAMN11866291

BioProject:

https://www.ncbi.nlm.nih.gov/bioproject/PRJNA544897

\section{LITERATURE CITED}

Adeniji, A. A., Aremu, O. S., and Babalola, O. O. 2019a. Selecting lipopeptide-producing, Fusarium-suppressing Bacillus spp.: Metabolomic and genomic probing of Bacillus velezensis NWUMFkBS10.5. MicrobiologyOpen 8:e00742.

Adeniji, A. A., and Babalola, O. O. 2018. Tackling maize fusariosis: In search of Fusarium graminearum biosuppressors. Arch. Microbiol. 200:1239-1255

Adeniji, A. A., Loots, D. T., and Babalola, O. O. 2019b. Bacillus velezensis: Phylogeny, useful applications, and avenues for exploitation. Appl. Microbiol. Biotechnol. 103:3669-3682.

Arkin, A. P., Stevens, R. L., Cottingham, R. W., Maslov, S., Henry, C. S., Dehal, P., Ware, D., Perez, F., Harris, N. L., and Canon, S. 2016. The DOE systems biology knowledgebase (KBase). BioRxiv:096354

Aziz, R. K., Bartels, D., Best, A. A., DeJongh, M., Disz, T., Edwards, R. A., Formsma, K., Gerdes, S., Glass, E. M., Kubal, M., Meyer, F., Olsen, G. J., Olson, R., Osterman, A. L., Overbeek, R. A., McNeil, L. K., Paarmann, D., Paczian, T., Parrello, B., Pusch, G. D., Reich, C., Stevens, R., Vassieva, O., Vonstein, V., Wilke, A., and Zagnitko, O. 2008. The RAST Server: Rapid annotations using subsystems technology. BMC Genomics 9:75.

Azizoglu, U. 2019. Bacillus thuringiensis as a biofertilizer and biostimulator: A mini-review of the little-known plant growth-promoting properties of Bt. Curr. Microbiol. 76:1379-1385.

Blin, K., Shaw, S., Steinke, K., Villebro, R., Ziemert, N., Lee, S. Y., Medema, M. H., and Weber, T. 2019. antiSMASH 5.0: Updates to the secondary metabolite genome mining pipeline. Nucleic Acids Res. 47:W81-W87.

Chehimi, S., Pons, A.-M., Sablé, S., Hajlaoui, M.-R., and Limam, F. 2010. Mode of action of thuricin S, a new class IId bacteriocin from Bacillus thuringiensis. Can. J. Microbiol. 56:162-167.

Christinet, L., Burdet, F. X., Zaiko, M., Hinz, U., and Zrÿd, J.-P. 2004. Characterization and functional identification of a novel plant 4,5-extradiol dioxygenase involved in betalain pigment biosynthesis in Portulaca grandiflora. Plant Physiol. 134:265-274.
Daas, M. S., Rosana, A. R. R., Acedo, J. Z., Douzane, M., Nateche, F., Kebbouche-Gana, S., and Vederas, J. C. 2018. Insights into the draft genome sequence of bioactives-producing Bacillus thuringiensis DNG9 isolated from Algerian soil-oil slough. Stand. Genomic Sci. 13:25.

Douriet-Gámez, N. R., Maldonado-Mendoza, I. E., Ibarra-Laclette, E., Blom, J., and Calderón-Vázquez, C. L. 2018. Genomic analysis of Bacillus sp. strain B25, a biocontrol agent of maize pathogen Fusarium verticillioides. Curr. Microbiol. 75:247-255.

Egidi, E., Wood, J. L., Mathews, E., Fox, E., Liu, W., and Franks, A. E. 2016. Draft genome sequence of Bacillus cereus LCR12, a plant growth-promoting rhizobacterium isolated from a heavy metal-contaminated environment. Genome Announce. 4:e01041-16.

Fusetti, F., Schröter, K. H., Steiner, R. A., van Noort, P. I., Pijning, T., Rozeboom, H. J., Kalk, K. H., Egmond, M. R., and Dijkstra, B. W. 2002. Crystal structure of the copper-containing quercetin 2,3-dioxygenase from Aspergillus japonicus. Structure 10:259-268.

Hao, Z., Yan, L., Liu, J., Song, F., Zhang, J., and Li, X. 2015. Extraction of antibiotic zwittermicin A from Bacillus thuringiensis by macroporous resin and silica gel column chromatography. Biotechnol. Appl. Biochem. 62:369-374.

Hollensteiner, J., Wemheuer, F., Harting, R., Kolarzyk, A. M., Diaz Valerio, S. M., Poehlein, A., Brzuszkiewicz, E. B., Nesemann, K., BrausStromeyer, S. A., Braus, G. H., Daniel, R., and Liesegang, H. 2017. Bacillus thuringiensis and Bacillus weihenstephanensis inhibit the growth of phytopathogenic verticillium species. Front. Microbiol. 7:2171.

Overbeek, R., Olson, R., Pusch, G. D., Olsen, G. J., Davis, J. J., Disz, T., Edwards, R. A., Gerdes, S., Parrello, B., Shukla, M., Vonstein, V., Wattam, A. R., Xia, F., and Stevens, R. 2014. The SEED and the Rapid Annotation of microbial genomes using Subsystems Technology (RAST). Nucleic Acids Res. 42:D206-D214.

Pruitt, K. D., Tatusova, T., Brown, G. R., and Maglott, D. R. 2012. NCBI Reference Sequences (RefSeq): Current status, new features and genome annotation policy. Nucleic Acids Res. 40:D130-D135.

Sansinenea, E., and Ortiz, A. 2012. Zwittermicin A: A promising aminopolyol antibiotic from biocontrol bacteria. Curr. Org. Chem. 16:978-987.

Steiner, R. A., Kalk, K. H., and Dijkstra, B. W. 2002. Anaerobic enzyme-substrate structures provide insight into the reaction mechanism of the copper-dependent quercetin 2,3-dioxygenase. Proc. Natl. Acad. Sci. U.S.A. 99:16625-16630.

Wattam, A. R., Davis, J. J., Assaf, R., Boisvert, S., Brettin, T., Bun, C., Conrad, N., Dietrich, E. M., Disz, T., Gabbard, J. L., Gerdes, S., Henry, C. S., Kenyon, R. W., Machi, D., Mao, C., Nordberg, E. K., Olsen, G. J., Murphy-Olson, D. E., Olson, R., Overbeek, R., Parrello, B., Pusch, G. D., Shukla, M., Vonstein, V., Warren, A., Xia, F., Yoo, H., and Stevens, R. L. 2017. Improvements to PATRIC, the allbacterial bioinformatics database and analysis resource center. Nucleic Acids Res. 45:D535-D542. 